\title{
The Existence and Attractivity of Solutions of an Urysohn Integral Equation on an Unbounded Interval
}

\author{
Mohamed Abdalla Darwish, ${ }^{1}$ Józef Banas, $^{2}$ and Ebraheem O. Alzahrani ${ }^{3}$ \\ ${ }^{1}$ Mathematics Department, Science Faculty for Girls, King Abdulaziz University, Jeddah, Saudi Arabia \\ ${ }^{2}$ Department of Mathematics, Rzeszów University of Technology, al. Powstańców Warszawy 8, 35-959 Rzeszów, Poland \\ ${ }^{3}$ Mathematics Depertment, Faculty of Science, King Abdulaziz University, Jeddah, Saudi Arabia \\ Correspondence should be addressed to Józef Banaś; jbanas@prz.edu.pl
}

Received 21 August 2013; Accepted 4 September 2013

Academic Editor: Mohammad Mursaleen

Copyright (c) 2013 Mohamed Abdalla Darwish et al. This is an open access article distributed under the Creative Commons Attribution License, which permits unrestricted use, distribution, and reproduction in any medium, provided the original work is properly cited.

We prove a result on the existence and uniform attractivity of solutions of an Urysohn integral equation. Our considerations are conducted in the Banach space consisting of real functions which are bounded and continuous on the nonnegative real half axis. The main tool used in investigations is the technique associated with the measures of noncompactness and a fixed point theorem of Darbo type. An example showing the utility of the obtained results is also included.

\section{Introduction}

The theory of nonlinear functional integral equations creates an important branch of the modern nonlinear analysis. The large part of that theory describes a lot of classical nonlinear integral equations such as nonlinear Volterra integral equations, Hammerstein integral equations, and Urysohn integral equations with solutions defined on a bounded interval (cf. $[1-4])$.

Nevertheless, more important and simultaneously, a more difficult part of that theory is connected with the study of solutions of the mentioned integral equations defined on an unbounded domain. Obviously, there are some known results concerning the existence of solutions of those integral equations in such a setting but, in general, they are mostly obtained under rather restrictive assumptions [2, 4-8].

On the other hand, the use of some tools of nonlinear analysis enables us to obtain several valuable results under less restrictive assumptions (cf. [1, 9-15]). It turns out that the technique of measures of noncompactness creates a very convenient tool for the study of the solvability of nonlinear functional integral equations of various types. It is caused by the fact that the approach to the study of solutions of those equations with the use of the technique of measures of noncompactness gives not only the possibility to obtain existence results, but also allows us to look for solutions of mentioned equations having some desired properties such as monotonicity, attractivity, and asymptotic stability (cf. [1619], for instance).

In the paper, we will use the above described approach associated with the technique of measures of noncompactness in order to obtain a result on the existence of solutions of a quadratic Urysohn integral equation. Applying the mentioned technique in conjunction with a fixed point theorem of Darbo type, we show that the equation in question has solutions defined, continuous, and bounded on the nonnegative real half axis $\mathbb{R}_{+}$which are uniformly attractive (asymptotic stable) on $\mathbb{R}_{+}$.

The results obtained in this paper generalize several results obtained earlier in numerous papers treating nonlinear functional integral equations, which were quoted above. Particularly, we generalize the results concerning the Urysohn or Hammerstein integral equations obtained in the papers $[9,10,20]$.

\section{Notation, Definitions, and Auxiliary Facts}

In this section, we establish some notations, and we collect auxiliary facts which will be used in the sequel. 
By the symbol $\mathbb{R}$ we denote the set of real numbers, while $\mathbb{R}_{+}$ stands for the half axis $[0, \infty)$. Further, assume that $(E,\|\cdot\|)$ is a given real Banach space with the zero element $\theta$. For $x \in E$ and for a fixed $r>0$, denote by $B(x, r)$ the closed ball centered at $x$ and with radius $r$. We write $B_{r}$ in order to denote the ball $B(\theta, r)$.

Moreover, if $X$ and $Y$ are nonempty subsets of $E$ and $\lambda \epsilon$ $\mathbb{R}$, then we denote by $X+Y, \lambda X$, the usual algebraic operations on sets. If $X$ is a subset of $E$ then the symbols $\bar{X}$ and Conv $X$ denote the closure and closed convex hull of $X$, respectively. Apart from this, we denote by $\mathfrak{M}_{E}$ the family of all nonempty and bounded subsets of $E$ and by $\mathfrak{N}_{E}$ its subfamily consisting of all relatively compact sets.

In what follows, we will accept the following definition of the concept of a measure of noncompactness [21].

Definition 1. A mapping $\mu: \mathfrak{M}_{E} \rightarrow \mathbb{R}_{+}$is said to be $a$ measure of noncompactness in $E$ if it satisfies the following conditions.

$\left(1^{\circ}\right)$ The family ker $\mu=\left\{X \in \mathfrak{M}_{E}: \mu(X)=0\right\}$ is nonempty and $\operatorname{ker} \mu \subset \mathfrak{N}_{E}$.

$\left(2^{\circ}\right) X \subset Y \Rightarrow \mu(X) \leq \mu(Y)$.

$\left(3^{\circ}\right) \mu(\bar{X})=\mu(\operatorname{Conv} X)=\mu(X)$.

$\left(4^{\circ}\right) \mu(\lambda X+(1-\lambda) Y) \leq \lambda \mu(X)+(1-\lambda) \mu(Y)$ for $\lambda \in[0,1]$.

$\left(5^{\circ}\right)$ If $\left(X_{n}\right)$ is a sequence of closed sets from $\mathfrak{M}_{E}$ such that $X_{n+1} \subset X_{n}$ for $n=1,2, \ldots$ and if $\lim _{n \rightarrow \infty} \mu\left(X_{n}\right)=0$, then the intersection set $X_{\infty}=\bigcap_{n=1}^{\infty} X_{n}$ is nonempty.

The family ker $\mu$ appearing in $1^{\circ}$ is called the kernel of the measure of noncompactness $\mu$.

Observe that the set $X_{\infty}$ from the axiom $5^{\circ}$ is a member of the family ker $\mu$. Indeed, since $\mu\left(X_{\infty}\right) \leq \mu\left(X_{n}\right)$ for any natural number $n$, we infer that $\mu\left(X_{\infty}\right)=0$. Consequently, $X_{\infty} \in$ ker $\mu$. This simple observation will be essential in our further investigations.

Now, we formulate a fixed point theorem of Darbo type which will be used further on [21].

Theorem 2. Let $\Omega$ be a nonempty, bounded, closed, and convex subset of the Banach space $E$, and let $F: \Omega \rightarrow \Omega$ be a continuous mapping. Assume that there exists a constant $k \in$ $[0,1)$ such that $\mu(F X) \leq k \mu(X)$ for any nonempty subset $X$ of $\Omega$. Then, $F$ has a fixed point in the set $\Omega$.

Remark 3. Denote by Fix $F$ the set of all fixed points of the operator $F$ belonging to $\Omega$. It can be easily seen [21] that the set Fix $F$ belongs to the family $\operatorname{ker} \mu$.

In what follows, we will work in the Banach space $B C\left(\mathbb{R}_{+}\right)$ consisting of all real functions $x=x(t)$ defined, continuous, and bounded on $\mathbb{R}_{+}$. This space will be endowed with the standard supremum norm

$$
\|x\|=\sup \left\{|x(t)|: t \in \mathbb{R}_{+}\right\} .
$$

Now, we recall the construction of a measure of noncompactness in the space $B C\left(\mathbb{R}_{+}\right)$which was introduced in [21]. To this end, fix a nonempty and bounded subset $X$ of the space $B C\left(\mathbb{R}_{+}\right)$and positive numbers $\varepsilon>0, T>0$. For $x \in X$, denote by $\omega^{T}(x, \varepsilon)$ the modulus of continuity of the function $x$ on the interval $[0, T]$; that is,

$$
\omega^{T}(x, \varepsilon)=\sup \{|x(t)-x(s)|: t, s \in[0, T],|t-s| \leq \varepsilon\} .
$$

Next, we put

$$
\begin{gathered}
\omega^{T}(X, \varepsilon)=\sup \left\{\omega^{T}(x, \varepsilon): x \in X\right\}, \\
\omega_{0}^{T}(X)=\lim _{\varepsilon \rightarrow 0} \omega^{T}(X, \varepsilon), \\
\omega_{0}(X)=\lim _{T \rightarrow \infty} \omega_{0}^{T}(X) .
\end{gathered}
$$

Further, for a fixed number $t \in \mathbb{R}_{+}$let us put

$$
\begin{gathered}
X(t)=\{x(t): x \in X\}, \\
\operatorname{diam} X(t)=\sup \{|x(t)-y(t)|: x, y \in X\} .
\end{gathered}
$$

Finally, let us consider the function $\mu$ defined on the family $\mathfrak{M}_{B C\left(\mathbb{R}_{+}\right)}$by the formula

$$
\mu(X)=\omega_{0}(X)+\limsup _{t \rightarrow \infty} \operatorname{diam} X(t) .
$$

It can be shown [21] that the function $\mu$ is a measure of noncompactness in the space $B C\left(\mathbb{R}_{+}\right)$. Moreover, the kernel ker $\mu$ of this measure consists of all nonempty and bounded subsets $X$ of $B C\left(\mathbb{R}_{+}\right)$such that functions from $X$ are locally equicontinuous on $\mathbb{R}_{+}$, and the thickness of the bundle formed by functions from $X$ tends to zero at infinity. This property in combination with Remark 3 permits us to characterize solutions of the integral equation considered in the sequel.

For further purposes, we introduce now the concept of attractivity (stability) of solutions of operator equations in the space $B C\left(\mathbb{R}_{+}\right)$. To this end, assume that $\Omega$ is a nonempty subset of the space $B C\left(\mathbb{R}_{+}\right)$. Moreover, let $F$ be an operator defined on $\Omega$ with values in $B C\left(\mathbb{R}_{+}\right)$. Let us consider the operator equation of the form

$$
x(t)=(F x)(t), \quad t \in \mathbb{R}_{+} .
$$

Definition 4. We say that solutions of (6) are attractive (or locally attractive) if there exists a ball $B\left(x_{0}, r\right)$ in the space $B C\left(\mathbb{R}_{+}\right)$such that $B\left(x_{0}, r\right) \cap \Omega \neq \emptyset$, and for arbitrary solutions $x=x(t), y=y(t)$ of (6) belonging to the set $B\left(x_{0}, r\right) \cap \Omega$ we have that

$$
\lim _{t \rightarrow \infty}(x(t)-y(t))=0 .
$$

In the case when limit (7) is uniform with respect to the set $B\left(x_{0}, r\right) \cap \Omega$, that is, when for each $\varepsilon>0$ there exists $T>0$ such that

$$
|x(t)-y(t)| \leq \varepsilon
$$

for all $x, y \in B\left(x_{0}, r\right) \cap \Omega$ being solutions of (6) and for any $t \geq T$, we will say that solutions of (6) are uniformly attractive (or asymptotically stable).

Notice that the previous definition comes from $[16,19]$. 


\section{Main Result}

We will consider the existence and asymptotic behaviour of solutions of the quadratic Urysohn integral equation having the form

$$
x(t)=a(t)+f(t, x(t)) \int_{0}^{\infty} u(t, s, x(s)) d s
$$

for $t \in \mathbb{R}_{+}$.

In our study, we will impose the following assumptions.

(i) $a \in B C\left(\mathbb{R}_{+}\right)$.

(ii) $f: \mathbb{R}_{+} \times \mathbb{R} \rightarrow \mathbb{R}$ is a continuous function, and the function $t \rightarrow f(t, 0)$ is a member of the space $B C\left(\mathbb{R}_{+}\right)$.

(iii) The function $f=f(t, x)$ satisfies the Lipschitz condition with respect to the second variable; that is, there exists a constant $k>0$ such that

$$
|f(t, x)-f(t, y)| \leq k|x-y|
$$

for $x, y \in \mathbb{R}_{+}$and $t \in \mathbb{R}_{+}$.

(iv) $u: \mathbb{R}_{+} \times \mathbb{R}_{+} \times \mathbb{R} \rightarrow \mathbb{R}$ is a continuous function, and there exists a continuous function $g: \mathbb{R}_{+} \times \mathbb{R}_{+} \rightarrow$ $\mathbb{R}_{+}$and a continuous, nondecreasing function $h$ : $\mathbb{R}_{+} \rightarrow \mathbb{R}_{+}$with $\lim _{\varepsilon \rightarrow 0} h(\varepsilon)=0$, such that

$$
|u(t, s, x)-u(t, s, y)| \leq g(t, s) h(|x-y|)
$$

for $t, s \in \mathbb{R}_{+}$and $x, y \in \mathbb{R}$.

(v) For each $t \in \mathbb{R}_{+}$, the functions $s \rightarrow g(t, s)$ and $s \rightarrow$ $|u(t, s, 0)|$ are integrable $\mathbb{R}_{+}$and

$$
\lim _{t \rightarrow \infty} \int_{0}^{\infty} g(t, s) d s=0 .
$$

Moreover, the function $t \rightarrow \int_{0}^{\infty}|u(t, s, 0)| d s$ is bounded on $\mathbb{R}_{+}$.

(vi) The following equalities hold:

$$
\begin{gathered}
\lim _{T \rightarrow \infty}\left\{\sup \left\{\int_{T}^{\infty} g(t, s) d s: t \in[0, T]\right\}\right\}=0, \\
\lim _{T \rightarrow \infty}\left\{\sup \left\{\int_{T}^{\infty}|u(t, s, 0)| d s: t \in[0, T]\right\}\right\}=0 .
\end{gathered}
$$

Let us observe that in view of assumptions (ii) and (v) we can define the following finite constants:

$$
\begin{gathered}
\bar{f}=\sup \left\{|f(t, 0)|: t \in \mathbb{R}_{+}\right\}, \\
\bar{g}=\sup \left\{\int_{0}^{\infty} g(t, s) d s: t \in \mathbb{R}_{+}\right\}, \\
\bar{u}=\sup \left\{\int_{0}^{\infty}|u(t, s, 0)| d s: t \in \mathbb{R}_{+}\right\} .
\end{gathered}
$$

Remark 5. It is worthwhile mentioning that in the theory of improper Riemann integral with a parameter there has been considered the concept of the uniform convergence of the improper integral with respect to that parameter (cf. [22]). In order to recall this concept, suppose that there is a given function $z(t, s)=z: \mathbb{R}_{+} \times \mathbb{R}_{+} \rightarrow \mathbb{R}$ such that the improper integral

$$
\int_{0}^{\infty} z(t, s) d s
$$

does exist for every fixed $t \in \mathbb{R}_{+}$.

We say that the integral (15) is uniformly convergent with respect to $t \in \mathbb{R}_{+}$if

$$
\lim _{T \rightarrow \infty} \int_{0}^{T} z(t, s) d s=\int_{0}^{\infty} z(t, s) d s
$$

uniformly with respect to $t \in \mathbb{R}_{+}$.

Equivalently (cf. [10]), the integral (15) is uniformly convergent with respect to $t \in \mathbb{R}_{+}$if

$$
\lim _{T \rightarrow \infty}\left\{\sup _{t \in \mathbb{R}_{+}} \int_{T}^{\infty} z(t, s) d s\right\}=0 .
$$

Let us observe that if integrals appearing in assumption (v), that is, the integrals

$$
\int_{0}^{\infty} g(t, s) d s, \quad \int_{0}^{\infty}|u(t, s, 0)| d s
$$

are uniformly convergent with respect to $t \in \mathbb{R}_{+}$, then the inequalities from assumption (vi) are satisfied (cf. [10]). Indeed, this conclusion follows easily from the inequalities

$$
\begin{gathered}
\sup _{t \in[0, T]} \int_{T}^{\infty} g(t, s) d s \leq \sup _{t \in \mathbb{R}_{+}} \int_{T}^{\infty} g(t, s) d s, \\
\sup _{t \in[0, T]} \int_{T}^{\infty}|u(t, s, 0)| d s \leq \sup _{t \in \mathbb{R}_{+}} \int_{T}^{\infty}|u(t, s, 0)| d s,
\end{gathered}
$$

which are valid for any $T>0$.

It may be also shown that the converse implications are, in general, not valid [10].

Remark 6. It can be also shown [10] that the requirements concerning the function $g(t, s)$ imposed in assumption (v) are independent, that is, there exist functions $g_{i}: \mathbb{R}_{+} \times$ $\mathbb{R}_{+} \rightarrow \mathbb{R}_{+}(i=1,2)$ such that for each $t \in \mathbb{R}_{+}$ there exist the integrals $\int_{0}^{\infty} g_{i}(t, s) d s(i=1,2)$ and such that the integral $\int_{0}^{\infty} g_{1}(t, s) d s$ is uniformly convergent but $\lim _{t \rightarrow \infty} \int_{0}^{\infty} g_{1}(t, s) d s \neq 0$ while $\lim _{t \rightarrow \infty} \int_{0}^{\infty} g_{2}(t, s) d s=0$, but the integral $\int_{0}^{\infty} g_{2}(t, s) d s$ is not uniformly convergent.

Now, we formulate our last assumption.

(vii) The inequality

$$
\|a\|+k \bar{g} r h(r)+k \bar{u} r+\bar{f} \bar{g} h(r)+\bar{f} \bar{u} \leq r
$$


has a positive solution $r_{0}$ such that

$$
k\left(\bar{g} h\left(r_{0}\right)+\bar{u}\right)<1
$$

Remark 7. Assume that $r_{0}>0$ satisfies the first inequality from assumption (vii); that is,

$$
\|a\|+k \bar{g} r_{0} h\left(r_{0}\right)+k \bar{u} r_{0}+\bar{f} \bar{g} h\left(r_{0}\right)+\bar{f} \bar{u} \leq r_{0}
$$

Then, we obtain

$$
k\left(\bar{g} h\left(r_{0}\right)+\bar{u}\right) \leq 1-\frac{\|a\|}{r_{0}}-\frac{\bar{f} \bar{g} h\left(r_{0}\right)}{r_{0}}-\frac{\bar{f} \bar{u}}{r_{0}} .
$$

Thus, the second inequality from assumption (vii) is satisfied provided at least one of the quantities $\|a\|, \bar{f} \bar{g} h\left(r_{0}\right)$, and $\bar{f} \bar{u}$ does not vanish.

Now, we are prepared to formulate our main result.

Theorem 8. Under assumptions (i)-(vii), (9) has at least one solution $x=x(t)$ in the space $B C\left(\mathbb{R}_{+}\right)$. Moreover, all solutions of (9) are uniformly attractive.

Proof. Consider the operator $U$ defined on the space $B C\left(\mathbb{R}_{+}\right)$ by the formula

$$
(U x)(t)=a(t)+f(t, x(t)) \int_{0}^{\infty} u(t, s, x(s)) d s, \quad t \in \mathbb{R}_{+} .
$$

Notice that in view of assumptions (i), (ii), (iv), and (v), the function $t \rightarrow(U x)(t)$ is well defined on the interval $\mathbb{R}_{+}$. We show that this function is continuous on $\mathbb{R}_{+}$. To this end, fix arbitrarily $T>0$ and $\varepsilon>0$. Next, take arbitrary numbers $t, s \in$ $[0, T]$ with $|t-s| \leq \varepsilon$. Then, in view of imposed assumptions we obtain

$$
\begin{aligned}
& |(U x)(t)-(U x)(s)| \\
& \leq|a(t)-a(s)| \\
& +\mid f(t, x(t)) \int_{0}^{\infty} u(t, \tau, x(\tau)) d \tau \\
& \quad-f(s, x(s)) \int_{0}^{\infty} u(t, \tau, x(\tau)) d \tau \mid \\
& +\mid f(s, x(s)) \int_{0}^{\infty} u(t, \tau, x(\tau)) d \tau \\
& \quad-f(s, x(s)) \int_{0}^{\infty} u(s, \tau, x(\tau)) d \tau \mid
\end{aligned}
$$

$$
\begin{aligned}
\leq & \omega^{T}(a, \varepsilon)+[|f(t, x(t))-f(t, x(s))| \\
& +|f(t, x(s))-f(s, x(s))|] \\
& \times \int_{0}^{\infty}|u(t, \tau, x(\tau))| d \tau \\
& +[|f(s, x(s))-f(s, 0)|+|f(s, 0)|] \\
& \times \int_{0}^{\infty}|u(t, \tau, x(\tau))-u(s, \tau, x(\tau))| d \tau \\
\leq & \omega^{T}(a, \varepsilon)+\left[k|x(t)-x(s)|+\omega_{\|x\|}^{T}(f, \varepsilon)\right]
\end{aligned}
$$$$
\times \int_{0}^{\infty}[|u(t, \tau, x(\tau))-u(t, \tau, 0)|
$$$$
+|u(t, \tau, 0)|] d \tau
$$$$
+[k|x(s)|+|f(s, 0)|]
$$$$
\times\left\{\int_{0}^{T}|u(t, \tau, x(\tau))-u(s, \tau, x(\tau))| d \tau\right.
$$$$
\left.+\int_{T}^{\infty}|u(t, \tau, x(\tau))-u(s, \tau, x(\tau))| d \tau\right\}
$$$$
\leq \omega^{T}(a, \varepsilon)+\left[k \omega^{T}(x, \varepsilon)+\omega_{\|x\|}^{T}(f, \varepsilon)\right]
$$$$
\times \int_{0}^{\infty}[g(t, \tau) h(|x(\tau)|)+|u(t, \tau, 0)|] d \tau
$$$$
+(k\|x\|+\bar{f})\left\{\int_{0}^{T} \omega_{\|x\|}^{T}(u, \varepsilon) d \tau\right.
$$$$
+\int_{T}^{\infty} \mid u(t, \tau, x(\tau))-u(t, \tau, 0)
$$$$
+u(t, \tau, 0)-u(s, \tau, 0)
$$$$
+u(s, \tau, 0)-u(s, \tau, x(\tau)) \mid d \tau\}
$$$$
\leq \omega^{T}(a, \varepsilon)+\left[k \omega^{T}(x, \varepsilon)+\omega_{\|x\|}^{T}(f, \varepsilon)\right]
$$$$
\times \int_{0}^{\infty}[g(t, \tau) h(\|x\|)+|u(t, \tau, 0)|] d \tau
$$$$
+(k\|x\|+\bar{f})\left\{\int_{0}^{T} \omega_{\|x\|}^{T}(u, \varepsilon) d \tau\right.
$$$$
+\int_{T}^{\infty}|u(t, \tau, x(\tau))-u(t, \tau, 0)| d \tau
$$$$
+\int_{T}^{\infty}|u(s, \tau, x(\tau))-u(s, \tau, 0)| d \tau
$$$$
+\int_{T}^{\infty}|u(t, \tau, 0)| d \tau
$$$$
\left.+\int_{T}^{\infty}|u(s, \tau, 0)| d \tau\right\}
$$ 


$$
\begin{aligned}
\leq & \omega^{T}(a, \varepsilon)+\left[k \omega^{T}(x, \varepsilon)+\omega_{\|x\|}^{T}(f, \varepsilon)\right] \\
& \times(\bar{g} h(\|x\|)+\bar{u})+(k\|x\|+\bar{f}) \\
& \times\left\{T \omega_{\|x\|}^{T}(u, \varepsilon)+2 \int_{T}^{\infty} g(t, s) h(\|x\|) d s\right. \\
& \left.\quad+\int_{T}^{\infty}|u(t, \tau, 0)| d \tau+\int_{T}^{\infty}|u(s, \tau, 0)| d \tau\right\},
\end{aligned}
$$

where we denoted

$$
\begin{aligned}
& \omega_{d}^{T}(f, \varepsilon)=\sup \{|f(t, x)-f(s, x)|: \\
& t, s \in[0, T],|t-s| \leq \varepsilon,|x| \leq d\}, \\
& \omega_{d}^{T}(u, \varepsilon)=\sup \{|u(t, \tau, x)-u(s, \tau, x)|: \\
& \quad t, s, \tau \in[0, T],|t-s| \leq \varepsilon,|x| \leq d\} .
\end{aligned}
$$

Obviously, in the previous performed calculations we should put $\|x\|$ in place of $d$.

Further, let us notice that in view of assumptions (ii) and (iv), the function $f$ is uniformly continuous on the set $[0, T] \times$ $[-\|x\|,\|x\|]$, while the function $u$ is uniformly continuous on the set $[0, T] \times[0, T] \times[-\|x\|,\|x\|]$. Hence, we derive that $\omega_{\|x\|}^{T}(f, \varepsilon) \rightarrow 0$ and $\omega_{\|x\|}^{T}(u, \varepsilon) \rightarrow 0$ as $\varepsilon \rightarrow 0$. Next, let us observe that based on assumption (vi) we can choose a number $T$ so large that the last terms in estimate (25), that is, the integrals

$$
\int_{T}^{\infty}|u(t, \tau, 0)| d \tau, \quad \int_{T}^{\infty}|u(s, \tau, 0)| d \tau,
$$

are sufficiently small. The same is also true with regard to the integral

$$
\int_{T}^{\infty} g(t, s) d s
$$

Thus, taking into account the all facts established above and estimate (25), we infer that the function $U x$ is continuous on the whole interval $[0, T]$ for each $T>0$ being big enough. This implies that $U x$ is continuous on the whole interval $\mathbb{R}_{+}$.

In what follows, we show that the function $U x$ is bounded on $\mathbb{R}_{+}$. Indeed, keeping in mind our assumptions, for an arbitrary fixed $t \in \mathbb{R}_{+}$, we get the following estimates:

$$
\begin{aligned}
|(U x)(t)| \leq & |a(t)|+|f(t, x(t))| \int_{0}^{\infty}|u(t, s, x(s))| d s \\
\leq & |a(t)|+[|f(t, x(t))-f(t, 0)|+|f(t, 0)|] \\
& \times \int_{0}^{\infty}[|u(t, s, x(s))-u(t, s, 0)|+|u(t, s, 0)|] d s \\
\leq & |a(t)|+(k\|x\|+\bar{f}) \\
& \times\left\{\int_{0}^{\infty} g(t, s) h(|x(s)|) d s+\int_{0}^{\infty}|u(t, s, 0)| d s\right\} .
\end{aligned}
$$

Hence, we obtain the following evaluation

$$
|(U x)(t)| \leq\|x\|+(k\|x\|+\bar{f})(\bar{g} h(\|x\|)+\bar{u}),
$$

which implies that the function $U x$ is bounded on $\mathbb{R}_{+}$. Combining this fact with the continuity of the function $U x$ on $\mathbb{R}_{+}$, we conclude that the operator $U$ transforms the space $B C\left(\mathbb{R}_{+}\right)$into itself.

Further, observe that from (30) we get

$$
\begin{aligned}
\|U x\| \leq & \|a\|+k \bar{g}\|x\| h(\|x\|) \\
& +k \bar{u}\|x\|+\bar{f} \bar{g} h(\|x\|)+\bar{f} \bar{u} .
\end{aligned}
$$

Linking the previous inequality with assumption (vii), we deduce that the operator $U$ maps the ball $B_{r_{0}}$ into itself, where $r_{0}>0$ is a number indicated in assumption (vii).

Now, let us take a nonempty subset $X$ of the ball $B_{r_{0}}$.

Fix numbers $\varepsilon>0$ and $T>0$ and choose an arbitrary function $x \in X$. Then, in virtue of estimate (25), for an arbitrary $t \in[0, T]$ we obtain

$$
\begin{aligned}
& \omega^{T}(U x, \varepsilon) \leq \omega^{T}(a, \varepsilon)+\left(\bar{g} h\left(r_{0}\right)+\bar{u}\right) k \omega^{T}(x, \varepsilon) \\
& +\left(\bar{g} h\left(r_{0}\right)+\bar{u}\right) \omega_{r_{0}}^{T}(f, \varepsilon)+\left(k r_{0}+\bar{f}\right) \\
& \times\left\{T \omega_{r_{0}}^{T}(u, \varepsilon)+2 h\left(r_{0}\right)\right. \\
& \times \sup \left[\int_{T}^{\infty} g(t, s) d s: t \in[0, T]\right] \\
& \left.+2 \sup \left[\int_{T}^{\infty}|u(t, \tau, 0)| d \tau: t \in[0, T]\right]\right\} .
\end{aligned}
$$

Hence, we derive the following estimate

$$
\begin{aligned}
\omega^{T}(U X, \varepsilon) \leq & \omega^{T}(a, \varepsilon)+\left(\bar{g} h\left(r_{0}\right)+\bar{u}\right) k \omega^{T}(X, \varepsilon) \\
+ & \left(\bar{g} h\left(r_{0}\right)+\bar{u}\right) \omega_{r_{0}}^{T}(f, \varepsilon)+\left(k r_{0}+\bar{f}\right) \\
& \times\left\{T \omega_{r_{0}}^{T}(u, \varepsilon)+2 h\left(r_{0}\right)\right. \\
& \times \sup \left[\int_{T}^{\infty} g(t, s) d s: t \in[0, T]\right] \\
& \left.+2 \sup \left[\int_{T}^{\infty}|u(t, \tau, 0)| d \tau: t \in[0, T]\right]\right\} .
\end{aligned}
$$

Further, keeping in mind the properties of the terms of the right hand side of the perviously obtained inequality, which 
were mentioned earlier (cf. assumptions (ii) and (iv)), we deduce that the following estimate holds

$$
\begin{aligned}
& \omega_{0}^{T}(U X) \\
& \leq k\left(\bar{g} h\left(r_{0}\right)+\bar{u}\right) \omega_{0}^{T}(X) \\
& +\left(k r_{0}+\bar{f}\right)\left\{\left[\int_{T}^{\infty}|u(t, \tau, 0)| d \tau: t \in[0, T]\right] 2 h\left(r_{0}\right)\right. \\
& \quad \times \sup \left[\int_{T}^{\infty} g(t, s) d s: t \in[0, T]\right] \\
& \left.+2 \sup \left[\int_{T}^{\infty}|u(t, \tau, 0)| d \tau: t \in[0, T]\right]\right\} .
\end{aligned}
$$

Consequently, in view of assumption (vi) we have

$$
\omega_{0}(U X) \leq k\left(\bar{g} h\left(r_{0}\right)+\bar{u}\right) \omega_{0}(X) .
$$

In what follows assume, as previously, that $X$ is a fixed nonempty subset of the ball $B_{r_{0}}$. Next, take arbitrary elements $x, y \in X$. Then, for an arbitrarily fixed $t \in \mathbb{R}_{+}$, in virtue of imposed assumptions, we obtain:

$$
\begin{aligned}
& |(U x)(t)-(U y)(t)| \\
& \leq \mid f(t, x(t)) \int_{0}^{\infty} u(t, s, x(s)) d s \\
& \quad-f(t, y(t)) \int_{0}^{\infty} u(t, s, x(s)) d s \mid \\
& +\mid f(t, y(t)) \int_{0}^{\infty} u(t, s, x(s)) d s \\
& \quad-f(t, y(t)) \int_{0}^{\infty} u(t, s, y(s)) d s \mid \\
& \leq|f(t, x(t))-f(t, y(t))| \int_{0}^{\infty}|u(t, s, x(s))| d s \\
& +|f(t, y(t))| \int_{0}^{\infty}|u(t, s, x(s))-u(t, s, y(s))| d s \\
& \quad+\quad|| f(t, y(t))-f(t, 0)|+| f(t, 0) \mid] \\
& \times \int_{0}^{\infty} g(t, s) h(|x(s)-y(s)|) d s \\
& \quad|x(t)-y(t)| \int_{0}^{\infty}[|u(t, s, x(s))-u(t, s, 0)| \\
& \quad+|u(t, s, 0)|] d s
\end{aligned}
$$

$$
\begin{aligned}
& \leq k|x(t)-y(t)|\left\{\int_{0}^{\infty} g(t, s) h(|x(s)|) d s\right. \\
& \left.\quad+\int_{0}^{\infty}|u(t, s, 0)| d s\right\} \\
& +(k|y(t)|+|f(t, 0)|) \\
& \quad \times \int_{0}^{\infty} g(t, s) h(|x(s)-y(s)|) d s \\
& \leq k\|x-y\| h(\|x\|) \int_{0}^{\infty} g(t, s) d s+k \bar{u}|x(t)-y(t)| \\
& \quad+(k\|y\|+\bar{f}) h(\|x-y\|) \int_{0}^{\infty} g(t, s) d s .
\end{aligned}
$$

Hence, we get

$$
\begin{aligned}
\operatorname{diam}(U X)(t) \leq & k \bar{u} \operatorname{diam} X(t) \\
& +\left\{2 k r_{0} h\left(r_{0}\right)+\left(k r_{0}+\bar{f}\right) h\left(2 r_{0}\right)\right\} \\
& \times \int_{0}^{\infty} g(t, s) d s .
\end{aligned}
$$

Taking into account assumption (v), from the previous inequality we derive the following one:

$$
\limsup _{t \rightarrow \infty} \operatorname{diam}(U X)(t) \leq k \bar{u} \limsup _{t \rightarrow \infty} \operatorname{diam} X(t) .
$$

Obviously, the previous inequality implies the following estimate:

$$
\begin{aligned}
& \limsup _{t \rightarrow \infty} \operatorname{diam}(U X)(t) \\
& \quad \leq k\left(\bar{g} h\left(r_{0}\right)+\bar{u}\right) \limsup _{t \rightarrow \infty} \operatorname{diam} X(t) .
\end{aligned}
$$

Finally, let us observe that by combining (35) and (39) we have

$$
\mu(U X) \leq k\left(\bar{g} h\left(r_{0}\right)+\bar{u}\right) \mu(X),
$$

where $\mu$ is the measure of noncompactness defined by formula (5).

In the last step of our proof, we show that the operator $U$ is continuous on the ball $B_{r_{0}}$. To this end, fix an arbitrary number $\varepsilon>0$ and take $x, y \in B_{r_{0}}$ such that $\|x-y\| \leq \varepsilon$. Then, from estimate (36) we obtain

$$
\|U x-U y\| \leq k \bar{g} h\left(r_{0}\right) \varepsilon+k \bar{u} \varepsilon+\left(k r_{0}+\bar{f}\right) \bar{g} h(\varepsilon) .
$$

The previous inequality in conjunction with assumption (iv) implies that the operator $U$ is a continuous self-mapping of the ball $B_{r_{0}}$.

Finally, using the above established facts and (40) and taking into account assumption (vii) and Theorem 2, we infer that the operator $U$ has at least one fixed point $x$ in the ball $B_{r_{0}}$. Obviously, every function $x=x(t)$ being a fixed point of the operator $U$, is a solution of (9). Moreover, keeping in mind Remark 3, we conclude that the set FixU of all fixed points 
of the operator $U$ belonging to the ball $B_{r_{0}}$ (equivalently: the set FixU of all solutions of (9) belonging to the ball $B_{r_{0}}$ ) is a member of ker $\mu$. Hence, in view of the description of the kernel ker $\mu$ given in Section 2 we infer that all solutions of (9) belonging to the ball $B_{r_{0}}$ are uniformly attractive (asymptotically stable).

The proof is complete.

It is worthwhile mentioning that the above result generalizes those obtained in $[3,5,8,10,17,20]$, among others.

Now, we are going to illustrate the result contained in Theorem 8 by an example.

Example 9. Let us consider the following quadratic Urysohn integral equation:

$$
x(t)=\frac{\alpha e^{t}}{1+e^{t}}+\beta \frac{t^{2}+\sin x(t)}{1+t^{2}} \int_{0}^{\infty} \frac{\sqrt[3]{t^{3 / 2}+x^{2}(s)}}{1+t+s^{2}} d s
$$

where $t \in \mathbb{R}_{+}$and $\alpha, \beta$ are positive constants.

Observe that this equation is a special case of (9) if we put

$$
\begin{gathered}
a(t)=\frac{\alpha e^{t}}{1+e^{t}}, \\
f(t, x)=\beta \frac{t^{2}+\sin x}{1+t^{2}}, \\
u(t, s, x)=\frac{\sqrt[3]{t^{3 / 2}+x^{2}}}{1+t+s^{2}} .
\end{gathered}
$$

It is easily to check that for the previous functions there are satisfied assumptions of Theorem 8 . Indeed, the function $a=$ $a(t)$ is an element of the space $B C\left(\mathbb{R}_{+}\right)$and $\|a\|=\alpha$. This means that there is verified assumption (i). Next, notice that $f$ is continuous on $\mathbb{R}_{+} \times \mathbb{R}$ and $f(t, 0)=\beta t^{2} /\left(1+t^{2}\right)$. Thus, the function $t \rightarrow f(t, 0)$ belongs to $B C\left(\mathbb{R}_{+}\right)$, and there is satisfied assumption (ii). Moreover, we have that $\bar{f}=\beta$. Apart from this, for $x, y \in \mathbb{R}$ and for $t \in \mathbb{R}_{+}$we obtain

$$
\begin{aligned}
|f(t, x)-f(t, y)| & \leq \beta \frac{|\sin x-\sin y|}{1+t^{2}} \\
& \leq \beta \frac{|x-y|}{1+t^{2}} \leq \beta|x-y| .
\end{aligned}
$$

This implies that the function $f(t, x)$ satisfies assumption (iii) with $k=\beta$.

Further, let us note that the function $u(t, s, x)$ is continuous on the set $\mathbb{R}_{+} \times \mathbb{R}_{+} \times \mathbb{R}$. For arbitrarily fixed $t, s \in \mathbb{R}_{+}$, and $x, y \in \mathbb{R}$ we obtain

$$
|u(t, s, x)-u(t, s, y)| \leq \frac{\left|\sqrt[3]{t^{3 / 2}+x^{2}}-\sqrt[3]{t^{3 / 2}+y^{2}}\right|}{1+t+s^{2}} .
$$

Hence, using the inequality (cf. [9])

$$
\left|\sqrt[3]{a+x^{2}}-\sqrt[3]{a+y^{2}}\right| \leq \sqrt[3]{(x-y)^{2}}
$$

which is satisfied for all $x, y \in \mathbb{R}$ and for any fixed $a, a \geq 0$, we obtain

$$
|u(t, s, x)-u(t, s, y)| \leq \frac{(x-y)^{2 / 3}}{1+t+s^{2}} .
$$

This implies that the function $u(t, s, x)$ satisfies assumption (iv) with $h(r)=r^{2 / 3}$ and $g(t, s)=1 /\left(1+t+s^{2}\right)$. Obviously, the function $h: \mathbb{R}_{+} \rightarrow \mathbb{R}_{+}$and is continuous and increasing on $\mathbb{R}_{+}$, while the function $g(t, s)$ transforms $\mathbb{R}_{+} \times \mathbb{R}_{+}$into $\mathbb{R}_{+}$ and is continuous on $\mathbb{R}_{+} \times \mathbb{R}_{+}$.

Moreover, we get

$$
\begin{aligned}
\int_{0}^{\infty} g(t, s) d s & =\lim _{A \rightarrow \infty} \int_{0}^{A} g(t, s) d s \\
& =\lim _{A \rightarrow \infty} \frac{1}{t+1} \int_{0}^{A} \frac{d s}{1+(s / \sqrt{1+t})^{2}} \\
& =\lim _{A \rightarrow \infty} \frac{\sqrt{1+t}}{1+t} \arctan \frac{A}{\sqrt{1+t}} \\
& =\frac{\sqrt{1+t}}{1+t} \cdot \frac{\pi}{2} .
\end{aligned}
$$

Thus, the function $g(t, s)$ is integrable over $\mathbb{R}_{+}$. Next, we obtain

$$
\lim _{t \rightarrow \infty} \int_{0}^{\infty} g(t, s) d s=\lim _{t \rightarrow \infty} \frac{\sqrt{1+t}}{1+t} \frac{\pi}{2}=0 .
$$

Apart from this, in view of (48) we can easily to obtain that

$$
\bar{g}=\sup \left\{\int_{0}^{\infty} g(t, s) d s: t \in \mathbb{R}_{+}\right\}=\frac{\pi}{2} .
$$

Further, let us fix arbitrarily a number $T>0$. Then, similarly as above, we get

$$
\begin{aligned}
\sup _{t \in[0, T]} & \int_{t}^{\infty} g(t, s) d s \\
& =\sup _{t \in[0, T]}\left\{\frac{\sqrt{1+t}}{1+t}\left(\frac{\pi}{2}-\arctan \frac{T}{\sqrt{t+1}}\right)\right\} \\
& \leq \frac{\pi}{2}-\arctan \frac{T}{\sqrt{T+1}} .
\end{aligned}
$$

This allows us to deduce the following equality:

$$
\lim _{T \rightarrow \infty}\left\{\sup _{t \in[0, T]} \int_{T}^{\infty} g(t, s) d s\right\}=0 .
$$

Finally, let us take into account the function

$$
u(t, s, 0)=|u(t, s, 0)|=\frac{\sqrt{t}}{1+t+s^{2}} .
$$

Calculating the indefinite integral of the function $u(t, s, 0)$, we obtain

$$
\int u(t, s, 0) d s=\frac{\sqrt{t^{2}+t}}{t+1} \arctan \frac{s}{\sqrt{t+1}} .
$$


Hence, we get

$$
\begin{aligned}
\int_{0}^{\infty}|u(t, s, 0)| d s & =\int_{0}^{\infty} u(t, s, 0) d s \\
& =\lim _{A \rightarrow \infty} \int_{0}^{A} u(t, s, 0) d s \\
& =\lim _{A \rightarrow \infty} \frac{\sqrt{t^{2}+t}}{t+1} \arctan \frac{A}{\sqrt{t+1}} \\
& =\frac{\sqrt{t^{2}+t}}{t+1} \frac{\pi}{2} .
\end{aligned}
$$

Hence, taking into account that

$$
\sup _{t \in \mathbb{R}_{+}} \frac{\sqrt{t^{2}+t}}{t+1}=1
$$

we derive the following equality:

$$
\bar{u}=\sup _{t \in \mathbb{R}_{+}} \int_{0}^{\infty}|u(t, s, 0)| d s=\frac{\pi}{2} .
$$

Now, fix arbitrarily a number $T>0$. Then, from (54) we get

$$
\int_{T}^{\infty}|u(t, s, 0)| d s=\frac{\sqrt{t^{2}+t}}{t+1}\left(\frac{\pi}{2}-\arctan \frac{T}{\sqrt{t+1}}\right) .
$$

This allows us to derive the following estimate:

$$
\sup _{t \in[0, T]} \int_{T}^{\infty}|u(t, s, 0)| d s \leq \frac{\pi}{2}-\arctan \frac{T}{\sqrt{T+1}} .
$$

Consequently, this yields that the following equality holds:

$$
\lim _{T \rightarrow \infty}\left\{\sup _{t \in[0, T]} \int_{T}^{\infty}|u(t, s, 0)| d s\right\}=0 .
$$

Next, observe that taking into account (48), (52), (55), and (60), we conclude that the functions $g(t, s)$ and $u(t, s, 0)$ satisfy assumptions (v) and (vi).

Now, we are coming to the last assumption of Theorem 8 , that is, assumption (vii). Notice, that in the case of our Equation (42), in view of estimates (44), (50), and (55), the first inequality from assumption (vii) has the form

$$
\alpha+\beta \cdot \frac{\pi}{2} r \cdot r^{2 / 3}+\beta \frac{\pi}{2} r+\beta \cdot \frac{\pi}{2} r^{2 / 3}+\beta \frac{\pi}{2} \leq r .
$$

Hence, after some simplification, we obtain

$$
\alpha+\frac{\pi}{2} \beta\left(r^{5 / 3}+r+r^{2 / 3}+1\right) \leq r .
$$

Thus, if for fixed positive $\alpha$ and $\beta$ there exists a number $r_{0}>0$ satisfying inequality (62), then the second inequality from assumption (vii) is automatically satisfied. Indeed, it is an immediate consequence of Remark 7. In such a case (42) has solutions belonging to the ball $B_{r_{0}}$ which are uniformly attractive.

For example, it is easy to check that if we take $\alpha=\beta=1 / 8$ then the number $r_{0}=4 / 5$ satisfies inequality (62). This means that (42) with $\alpha=\beta=1 / 8$ has solutions belonging to the ball $B_{4 / 5}$ being uniformly attractive.

\section{Acknowledgment}

This project was funded by the Deanship of Scientific Research (DSR), King Abdulaziz University, Jeddah, under Grant no. (470/363/1433). The authors, therefore, acknowledge with thanks DSR technical and financial support.

\section{Conflict of Interests}

The authors declare that there is no conflict of interests in the submitted paper.

\section{References}

[1] J. Banaś and K. Sadarangani, "Compactness conditions in the study of functional, differential, and integral equations," Abstract and Applied Analysis, vol. 2013, Article ID 819315, 14 pages, 2013.

[2] K. Deimling, Nonlinear Functional Analysis, Springer, Berlin, Germany, 1985.

[3] M. A. Krasnosel'skii, P. P. Zabrejko, J. I. Pustylnik, and P. I. Sobolevskii, Integral Operators in Spaces of Summable Functions, Nordhoff, Leyden, Mass, USA, 1976.

[4] P. P. Zabrejko, A. I. Koshelev, M. A. Krasnosel'skii, S. G. Mikhlin, L. S. Rakovschik, and V. J. Stetsenko, Integral Equations, Nordhoff, Leyden, Mass, USA, 1975.

[5] I. J. Cabrera and K. B. Sadarangani, "Existence of solutions of a nonlinear integral equation on an unbounded interval," Dynamic Systems and Applications, vol. 18, no. 3-4, pp. 551-570, 2009.

[6] C. Corduneanu, Intergral Equations and Applications, Cambridge University Press, Cambridge, UK, 1991.

[7] N. Dunford and J. T. Schwartz, Linear Operators I, International Publishing, Leyden, The Netherlands, 1963.

[8] D. O'Regan and M. Meehan, Existence Theory for Nonlinear Integral and Integrodifferential Equations, Kluwer Academic, Dordrecht, The Netherlands, 1998.

[9] R. P. Agarwal, J. Banaś, K. Banaś, and D. O’Regan, “Solvability of a quadratic Hammerstein integral equation in the class of functions having limits at infinity," Journal of Integral Equations and Applications, vol. 23, no. 2, pp. 157-181, 2011.

[10] J. Banaś and L. Olszowy, "On solutions of a quadratic Urysohn integral equation on an unbounded interval," Dynamic Systems and Applications, vol. 17, no. 2, pp. 255-270, 2008.

[11] M. A. Darwish and J. Henderson, "Nondecreasing solutions of a quadratic integral equation of Urysohn-Stieltjes type," The Rocky Mountain Journal of Mathematics, vol. 42, no. 2, pp. 545566, 2012.

[12] M. A. Darwish and K. Sadarangani, "Nondecreasing solutions of a quadratic Abel equation with supremum in the kernel," Applied Mathematics and Computation, vol. 219, no. 14, pp. 7830-7836, 2013.

[13] M. Gil and S. Wedrychowicz, "Schauder-Tychonoff fixed-point theorem in the theory of superconductivity," Journal of Function Spaces and Applications, vol. 2013, Article ID 692879, 12 pages, 2013.

[14] L. Olszowy, "Fixed point theorems in the Fréchet space $C\left(\mathbb{R}_{+}\right)$ and functional integral equations on an unbounded interval," Applied Mathematics and Computation, vol. 218, no. 18, pp. 9066-9074, 2012. 
[15] L. Olszowy, "Nondecreasing solutions of a quadratic integral equation of Urysohn type on unbounded interval," Journal of Convex Analysis, vol. 18, no. 2, pp. 455-464, 2011.

[16] B. C. Dhage and V. Lakshmikantham, "On global existence and attractivity results for nonlinear functional integral equations," Nonlinear Analysis: Theory, Methods \& Applications A, vol. 72, no. 5, pp. 2219-2227, 2010.

[17] A. Aghajani and N. Sabzali, "Existence and local attractivity of solutions of a nonlinear quadratic functional integral equation," Iranian Journal of Science and Technology, Transaction A, vol. 36, no. 4, pp. 453-460, 2012.

[18] M. A. Darwish, "Monotonic solutions of a convolution functional-integral equation," Applied Mathematics and Computation, vol. 219, no. 22, pp. 10777-10782, 2013.

[19] X. Hu and J. Yan, "The global attractivity and asymptotic stability of solution of a nonlinear integral equation," Journal of Mathematical Analysis and Applications, vol. 321, no. 1, pp. 147156, 2006.

[20] R. Stańczy, "Hammerstein equations with an integral over a noncompact domain," Annales Polonici Mathematici, vol. 69, no. 1, pp. 49-60, 1998.

[21] J. Banaś and K. Goebel, Measures of Noncompactness in Banach Spaces, vol. 60 of Lecture Notes in Pure and Applied Mathematics, Marcel Dekker, New York, NY, USA, 1980.

[22] G. M. Fichtenholz, Differential and Integral Calculus, vol. 2, Wydawnictwo Naukowe PWN, Warsaw, Poland, 2007, (Polish). 


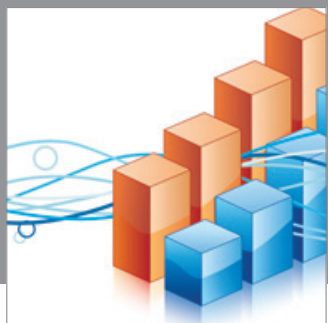

Advances in

Operations Research

mansans

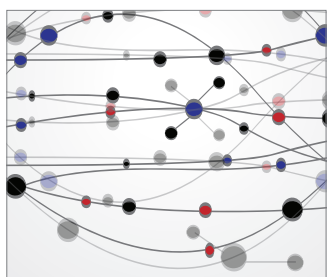

The Scientific World Journal
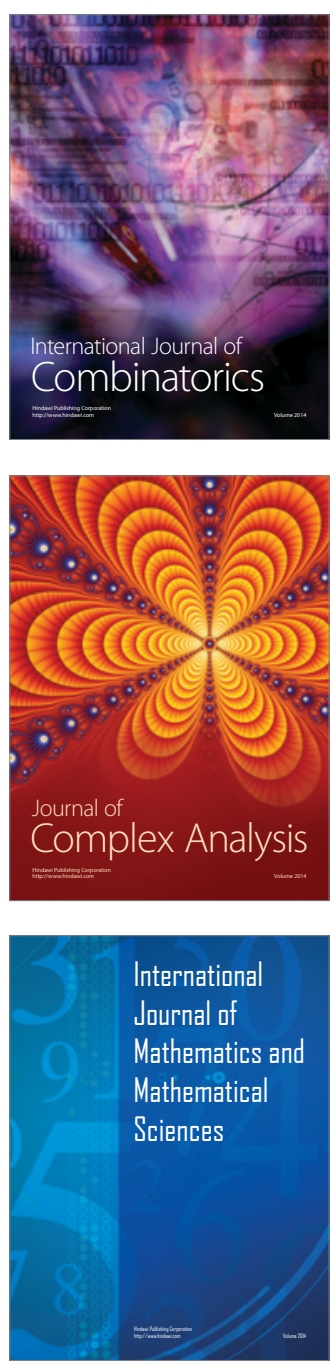
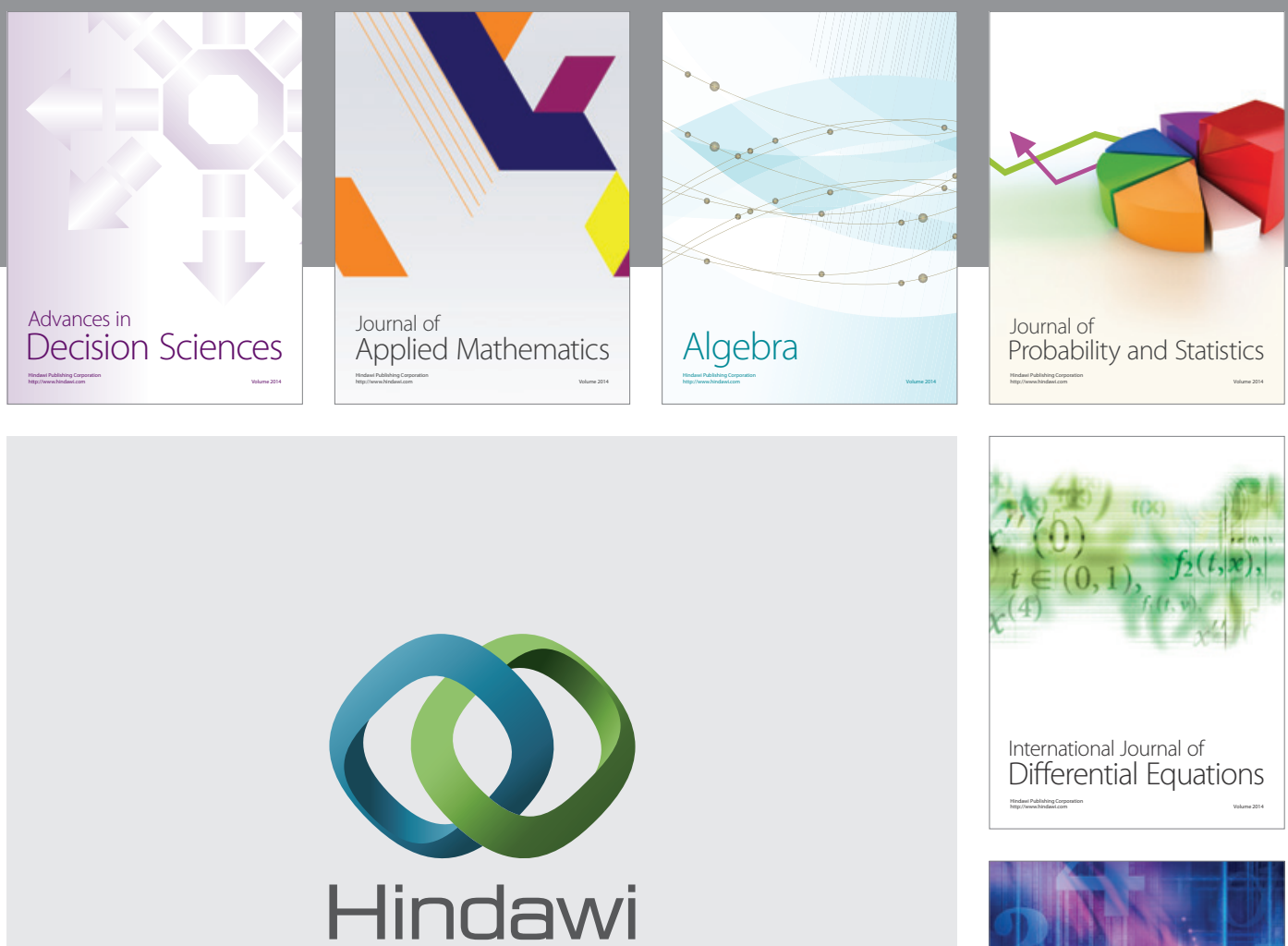

Submit your manuscripts at http://www.hindawi.com
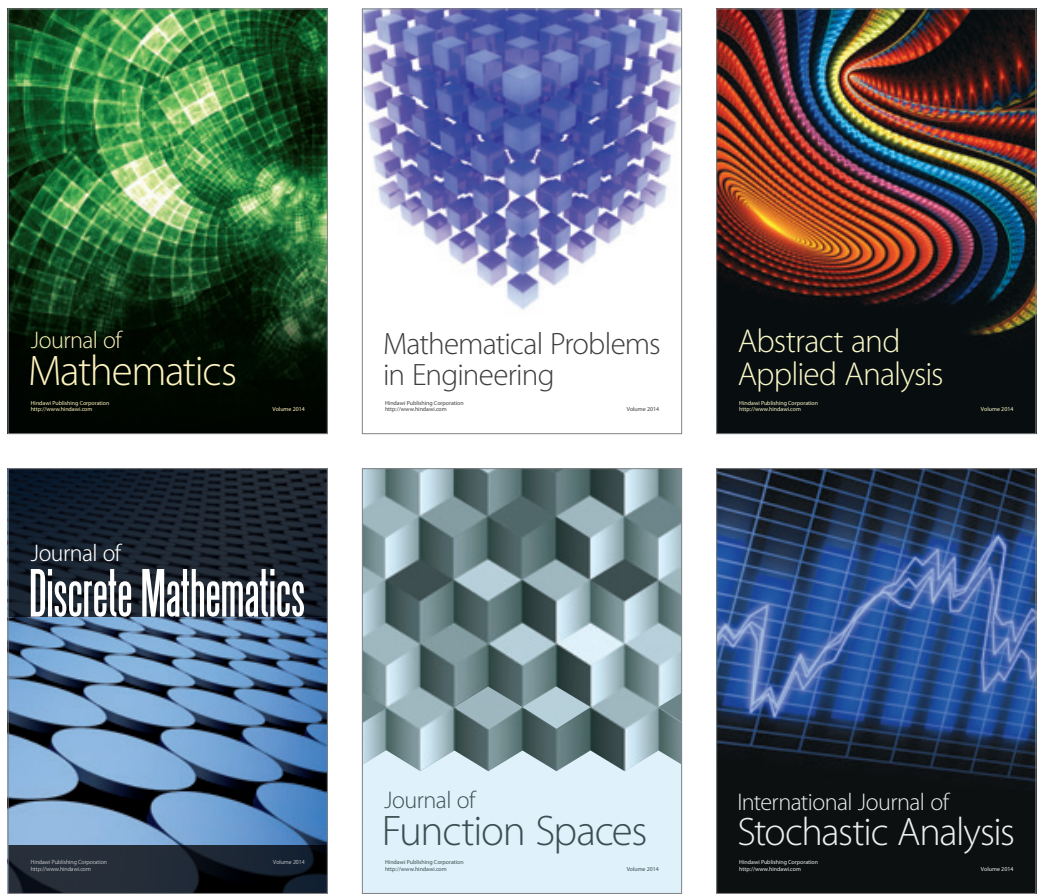

Journal of

Function Spaces

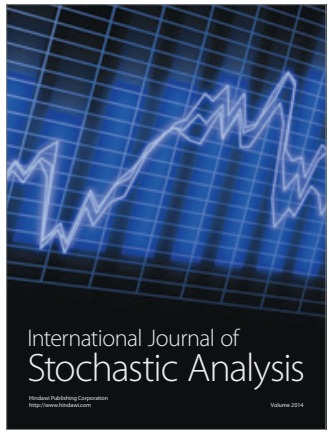

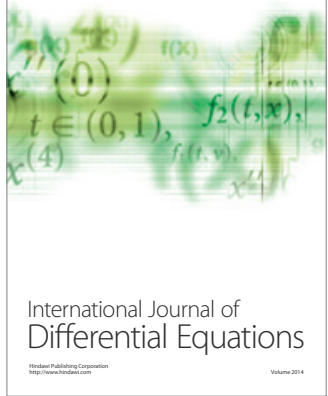
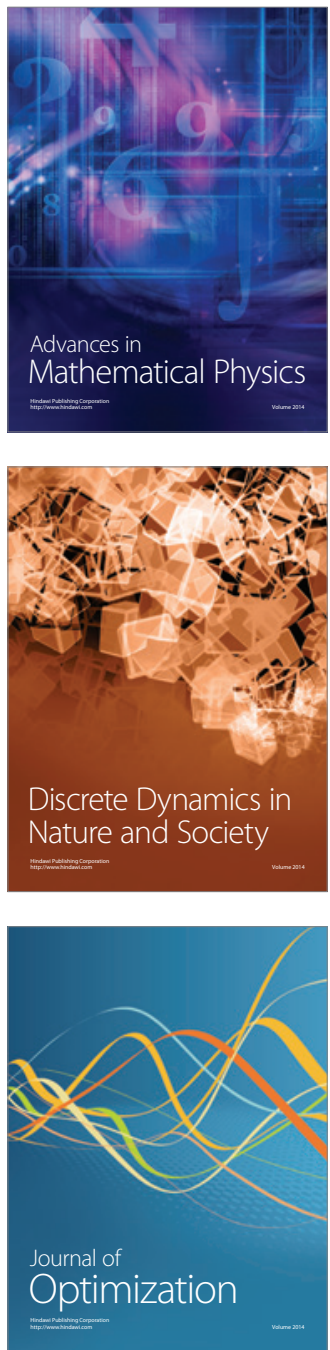